\title{
ENGAGEMENT AND USAGE PATTERNS OF A MOBILE APPLICATION TO MONITOR DISEASE AND TREATMENT ADHERENCE IN PATIENTS WITH ASTHMA
}

\author{
Rute Almeida ${ }^{1}$, Cristina Jácome $^{1}$, Pedro Vieira-Marques ${ }^{1}$, Ana Pereira ${ }^{1}$, Ana Sá-Sousa ${ }^{1}$, \\ Rita Amaral ${ }^{1,2}$, José Valente ${ }^{1,3}$ and João Almeida Fonseca ${ }^{1,3}$ \\ ${ }^{1}$ CINTESIS - Center for Health Technology and Services Research \& MEDCIDS - Department of Community Medicine, \\ Health Information and Decision, Faculty of Medicine, University of Porto, Porto, Portugal \\ ${ }^{2}$ Porto Health School, Polytechnic Institute of Porto, Porto, Portugal \\ ${ }^{3}$ MEDIDA - Serviços em Medicina, EDucação, Investigação, Desenvolvimento e Avaliação, LDA, Porto, Portugal
}

\begin{abstract}
Inhaled therapies are the cornerstone of effective asthma treatment and adequate inhaled medication adherence (IMA) is critical. mHealth technologies have shown to be promise for asthma self-management, including IMA improvement. InspirerMundi is a mobile application designed to support self-management of patients with asthma. It aims to transform the adherence to treatment into a positive experience through gamification and social interaction while allowing for ubiquitous verified IMA monitoring. The app includes an image-based inhaler usage detection tool, tools for reporting symptoms and burden of the asthma (disease monitoring) and Game \& Peer support features. Still, the effectiveness of these tools depends on a regular app use and a real-life assessment of patient engagement and pattern of use is needed.

This work evaluates the patient engagement, usage, and acceptance of InspirerMundi app during a real-world multicentre feasibility study. The app use was recommended for a 4-months period to 77 participants with persistent asthma. From those, 72 installed the app, with $67 \%$ of them beginning to use both the inhaler usage detection tool and the disease monitoring components within the first week. Over $95 \%$ used it more than once, with the period of usage (from first use to last registered monitoring in 2018) being over 30 days for almost $70 \%$ of the users, and over 90 days for around $35 \%$. Nevertheless, the usage rate (ratio of the number of days with app usage and the period of use) had a median value of 0.6 and was above $75 \%$ for only $35 \%$ of the users, revealing room for improvement.

In general, the users started to use the app features right after installation and the usage patterns and retention rates indicate that InspirerMundi is well accepted among patients with asthma.
\end{abstract}

\section{KEYWORDS}

mHealth, User Engagement, Medication Adherence, Asthma

\section{INTRODUCTION}

Recent technology developments have been promoting patient empowerment for self-care more than ever before. The ubiquitous presence of smartphones and tablets, at increasingly low cost and computational power, create a clear opportunity for the development of customized mobile Health (mHealth) solutions for self-monitoring and management of chronic diseases. Solutions taking advantage of the embedded sensors of smart devices, with no external accessories, are even more attractive as they are easy to integrate into everyday lives and to disseminate without additional costs for patients.

Asthma is an increasing incidence, high-burden, chronic obstructive respiratory disease, starting most often in early life and affecting 434M individuals worldwide (Forum of International Respiratory Societies, 2017). The long-term goals of asthma management are to reduce symptoms and the severity and frequency of exacerbations, to which those patients are prone to (GINA, 2020). Inhaled therapies are the cornerstone of effective asthma treatment and adequate inhaled medication adherence (IMA) is critical. Insufficient IMA is associated with poor symptom control, exacerbations, emergency department visits, and hospitalizations, leading to disease progression, additional social burden and health costs (Antoniu, 2010; Doz et al., 2013). 
Adherence to medication for chronic diseases has reported rates from 15\% to 70\% (Engelkes et al., 2015; Bender, 2016). Considering the impact and the strong evidence showing that poor IMA is a major obstacle to reduce that burden, the development of effective strategies to improve IMA is critical. Recognizing this problem, key stakeholders stated the urge of putting IMA on the EU policy agenda (van Boven et al., 2017).

To improve IMA, we need to measure it objectively. Research shows that clinicians cannot accurately estimate patient adherence and that self-reported adherence to treatments tends to be fictionally high (by more than 50\%) (Ose et al., 2012; Bender, 2016; Ferreira et al., 2019; Jácome et al., 2019). The objective measurement of IMA is difficult, and several attempts have been made to make it more effective. Electronic monitoring devices that register IMA by logging the date and time an inhaler is actuated gained some acceptance, providing detailed information about patterns of medication use, gained some acceptance (Chan $e t$ al., 2015). Randomized trials in asthma demonstrated that these devices have benefits on IMA (up to 84\%), but they were not sustained over long periods of time (Morton et al., 2017). Also, their wide dissemination is difficult and costly. Implementation of low-cost, ubiquitous solutions for measuring IMA, easy to disseminate and accept in healthcare services, would impact large numbers of patients and are therefore warranted.

mHealth technologies proved promising for asthma self-management, including IMA improvement, allowing the combination of data gathering and computation, gamification elements for user engagement and support to behavior change (Deterding et al., 2011; Marcano Belisario et al., 2013; Haase, Farris and Dorsch, 2017; Sardi, Idri and Fernández-Alemán, 2017), although, they generally do not take full advantage of the available technology (Tinschert et al., 2017). Gaining and maintaining a person's awareness and attention is the first step in promoting behavior change, through the addition of elements of fun together with peer support to promote behavior change (DeSmet et al., 2014), namely on IMA (Bousquet et al., 2017; Kosse et al., 2017; Miller et al., 2017). With that in mind, the InspirerMundi app (Figure 1) was conceived as an attempt to transform the adherence to treatment into a positive experience through gamification and social interaction, while allowing for ubiquitous verified IMA monitoring. This work evaluates the patient engagement, usage and acceptance of the app during a real-world multicentre feasibility study.

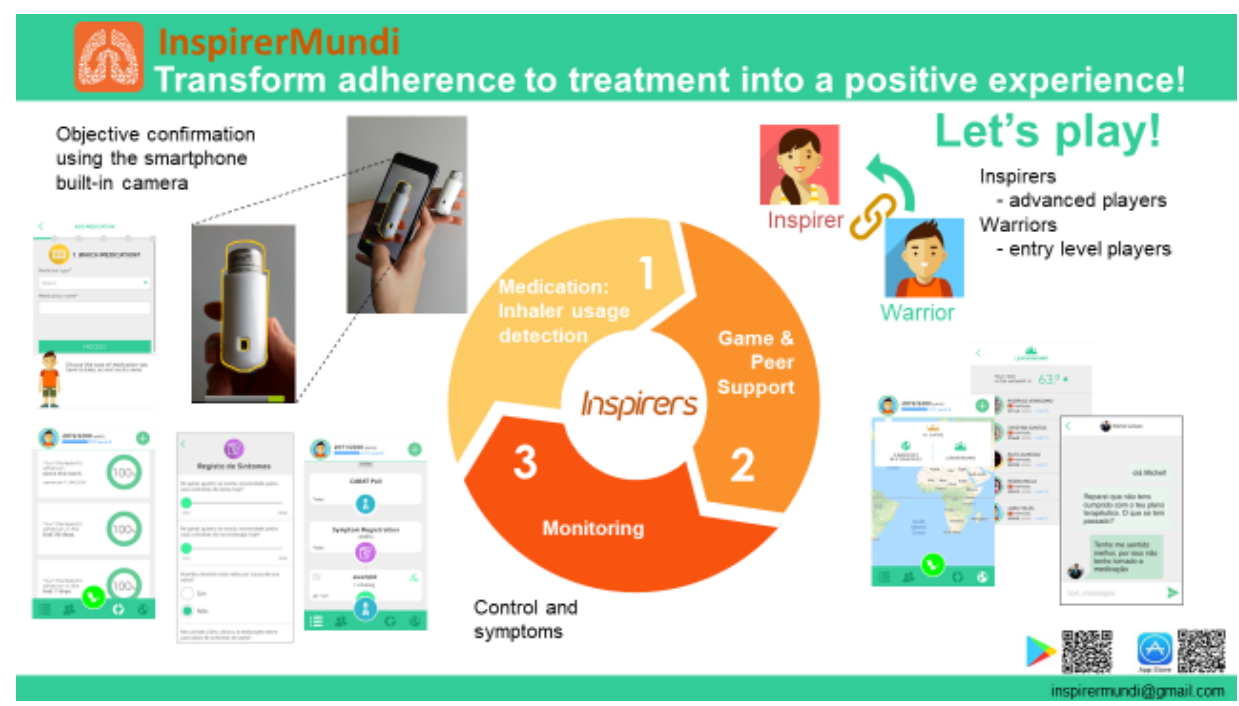

Figure 1. InspireMundi components

\section{METHODS AND DATA}

InspirerMundi aims to promote beneficial behavior changes in asthma patients' daily routine, with immediate and enjoyable feedback, and the delivery of easy-to-disseminate measures of IMA. The development phases have been previously described (Jácome et al., 2017). The app currently integrates different components regarding: 1. Medication, allowing to schedule alarms and including an image-based inhaler usage detection 
tool, 2. Game \& Peer support features and 3. Monitoring, to report asthma symptoms and burden (Figure 1). The functioning of the app is illustrated in videos available at the YouTube Chanel (InspirerMundi, 2020).

To address the need for objective IMA measures, the Inhaler detection tool uses the embedded mobile camera to capture a short video stream of the inhaler presented by the patients. Each frame is processed to confirm the type of inhaler (out of 6 available) using a double template, one with the inhaler outline and other with the counter outline. For inhalers without a correspondent template on the App, as well as for other types of medication, the usage detection module just collected a photo of the medication. Along with the confirmation of the type of inhaler, the app has a character recognition tool that can be used to assess the number of remaining doses for any inhaler providing a number-based dose counter (Vieira-Marques et al., 2020)

As is common in other gamified products, the app makes use of several game mechanics like points, badges and a leaderboard to engage the user. These are part of the game \& peer support component dynamics. Points can be earned using app features, except for unplanned use of reliever medication. Reaching certain goals is rewarded with virtual badges. By getting to higher game levels the user earns the possibility of evolving from Warrior, a beginner player, to Inspirer, a higher-grade player that has the possibility to socially influence others. Each Warrior can associate himself to an Inspirer, seeking peer support; allowing to exchange messages. These features enable Inspirers to stimulate Warriors to be compliant with the therapeutic plan. Users can see their position on the game leaderboard and visualize the network of influence on a world map.

In addition to the introduction of the therapeutic plan (medication name, dose, treatment duration, dosage, and schedule, when applicable), the app allows to register intakes, both for scheduled (including data from the Inhaler detection tool) and reliever medication. Reminders are generated to help the user to follow the plan. Asthma symptom control and burden are monitored through patient-reported outcomes measures, including- daily and weekly questionnaires, and the CARAT-Control of Allergic Rhinitis and Asthma Test, which is presented to the user on the onboarding day, and repeated on personalized intervals of 1,2 or 4 (default) weeks.

Introducing a therapeutic plan, registering medication intakes, and answering questionnaires are crucial to the correct use of the App, while actions related to the social component are optional. The mobile application was developed in native Swift language for iOS and Java for Android. In the web component, Drupal 7 was used as the content management system, as it is known to have a powerful ability to support Web services, and data was stored in a MySQL database. The use of the InspirerMundi App was assessed during 2018 using iOS versions 1 and 1.1 and Android versions 1.0.x, available at the mobile application stores under Health and Fitness category. These versions included templates for only 6 types of inhalers (Diskus/Accuhaler, Ellipta, Flutiform, Novolizer/Genuair, Spiromax, Turbohaler), which were the most used in Portugal.

Data from the real-world multicentre prospective observational feasibility study mInspirers 2018 study were analyzed (Jácome et al., 2019). It enrolled 77 adults and adolescents ( $>=13$ years old) with a previous medical diagnosis of persistent asthma, having an active prescription for an inhaled controller medication and followed by clinicians from the INSPIRERS group. The study did not interfere with the prescribed medications and all inhalers were admitted, even if their template was not included in the app. Patients were recruited in the first semester of 2018 on an initial face-to-face visit (T0) at one of 24 hospital care centers (Allergy, Pediatrics, Pulmonology outpatient clinics) in Portugal. The study was approved by the ethics committees of all participating centers. Eligible patients were approached by physicians during medical visits and informed and agreed to participate adults signed a consent form will adolescents signed an assent form, and a parental consent form was also obtained. They were invited to download and use the InspirerMundi app during at least a four months period. In T0 was collected data from patient and physician, including physician-reported information on prescribed control inhaled medications). Additionally, the patients received three follow-up phone interviews, at one week (T1, when additional support in App install and onboarding was given, if required), at one month (T2) and at four months (T3). Data were collected on: demographics, patients' opinions on mobile applications, satisfaction with InspirerMundi (global and regarding concerning different specific components, integration of functionalities, ease of use, and confidence; using a 5-item scale from 1, lower, to 5 , highest), willingness to use and recommend the app, as well as the perceived impact of use. Data from the app database collected up to the end 2018, including a minimum of six months after inclusion in the study, were retrieved. To validate the inhaler introduced in the therapeutic plan by the user, images captured using the image-based inhaler usage detection tool were distributed by 16 reviewers, who visually checked for validity (inhaler visible in the photo and agreement with the app registered inhaler type). 
The day of T0 (patient inclusion in the study) was defined as day 1 and used as a reference for engagement metrics. Onboarding, defined as the day of user creation in the app (first use), as well as the dates of use of specific features were included in the analysis. A user was considered active from onboarding until the last date of registered medication use or answered questionnaire in 2018. The period of use was defined as the number of days in between. A day with app usage is a day with at least one registered medication use or answered questionnaire. The usage rate was taken as the ratio between the number of days with app usage and the period of use. The arithmetic mean and standard deviation (mean[SD]) were considered to summarize engagement metrics, with frequency counts and median values also presented when most relevant. MATLAB 2018a) was used to perform statistical analysis.

\section{RESULTS}

From the 77 recruited participants, 72 (94\%) used the InspirerMundi app at least once. Three quarters $(\mathrm{n}=53)$ installed the Android version. From the app users, 57\% were female and ages ranged from 13 to 70 years old (27[12.7]), with $1 / 3$ under 18 and only $3 \%$ over 50 years old. Higher education degree was reported by $21 \%$ and high school degree by $44 \%$. Daily use of apps was reported by $82 \%$ of the users, $75 \%$ several times a day, download and use of health/fitness Apps by 54\%, but only one had previously used an asthma related App.

Onboarding occurred within the first week after inclusion for 57 (79\%) of the users (5.3[10.7] days from inclusion to onboarding). Figure 2 presents active app users per study week. More than $95 \%$ of the users used the app more than once and 65\% used the App for more than 10 days. The 30-day retention rate (considering all users) and 90-day retention rate (with respect to the previous 90 -days) were $70 \%$ and $50 \%$, respectively. The app usage from onboarding up to six months after inclusion ( 2 months after the 4-month of use requested by the study period) are displayed in Figure 3, sorted by use period. Of notice is the fact that some users did not use the app immediately after onboarding and initial weeks can also have no days with usage. Use patterns differed across users and the number of days between consecutive App uses ranged from zero to 87 . Nevertheless, more than $80 \%$ of consecutive app uses were on following days, as illustrated in Figure 4 . The usage rate had a median value of 0.6 (the distribution is presented in Figure 4.b). The period of use was longer than 30 days for almost 70\% of the users and longer than 90 days for around 35\% (67.3[60.4] days). More than $20 \%$ of the users used the app after day 120, i.e. after the requested use period within the study.

By the end of the first week of use, $74 \%$ of the users had introduced at least one medication, while $67 \%$ also answered at least one questionnaire. The proportion of users with at least a medication and a questionnaire increased to $74 \%$ by the end of the second week. Three users inserted medication, but did not answer any questionnaire, five answered at least one questionnaire, but did not insert any medication, while three did not use any of the two components. The median number of days with use of the medication or questionnaire modules was 30 days (38[39]), and the maximum was 160 days, with 47 (65\%) of the users returning to the app more than 10 times to use these features.

A total of 245 medications were inserted, 53 of which were reliever medications (repeating allowed). From the 138 entries for inhaled medication, 53 correspond to medication renew (same medication). Also, 38 entries were for reliever medication, 5 entries (4 different users) had the wrong type of medication chosen and one user indicated all 3 medications in the same entry (medication registration errors). The Add medication tool was used to insert control medication by 64 (88.9\%) of the users (Table 1). Up to 846 medication intakes were registered per user (100[140] intakes/user). Considering only users that inserted control medications, 90\% registered at least one intake, almost 70\% registered intakes in over 75\% of days with App usage (days in which they used the App to use medication or questionnaire modules) and 31\% registered intakes in all days with App usage. In the validation of the inhaler type 1251 images were reviewed from the first study participants (18 at the time of analysis). An inhaler was visible and identifiable in $84 \%$ of the images and it was in agreement with the user's choice in $96 \%$ of them. 
a) Onboarding

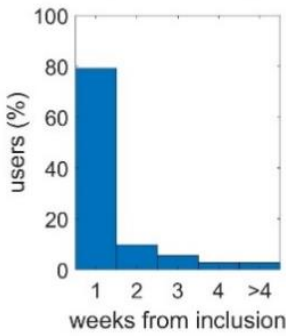

b) Active users

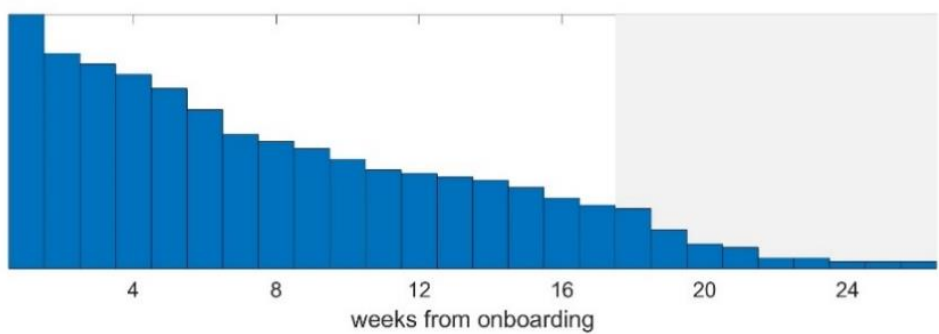

Figure 2. Panel a): Onboarding week (i.e. week of first use) from inclusion. Panel b: active users per week from onboarding (users with usage in that or posterior weeks) (the shaded area in grey identifies weeks after the four-month period of use requested by the study)

A total of 1634 questionnaires were answered by 66 users (92\%), distributed as indicated in Table 2. Respectively $63 \%$ and $42 \%$ of the users answered the first daily / weekly questionnaire on the onboarding day, as suggested. Globally, the compliance with questionnaires answering, accounting for the period of use and periodicity, was $62 \%$ for daily and $21 \%$ for weekly questionnaires (58[31] and 18[18] per user, respectively). For $65 \%$ of the users, the first CARAT was answered on the onboarding day. Those with at least two answers, had a mean time interval of 4.7 weeks between CARAT responses. The number of answered CARAT was equal or above the requested minimum (one at each four weeks for the period of app use) for $23 \%$ of the users, with around $60 \%$ missing just one questionnaire.

Regarding the social support component, five Inspirers and 22 Warrior invitations were formulated by 14 (19\%) study participants. Seven participants accepted to be a Warrior of other users (not necessarily participating in the study), while nine become Inspirers for someone else. Overall, only 10 (14\%) were an active part of a social network of influence.

Satisfaction with InspirerMundi was reported as a five-item scale of agreement level as summarized in Table 3. Except for the social support component, most users reported to be highly satisfied with all other app features (score of 4-5), both in T2 and T3.

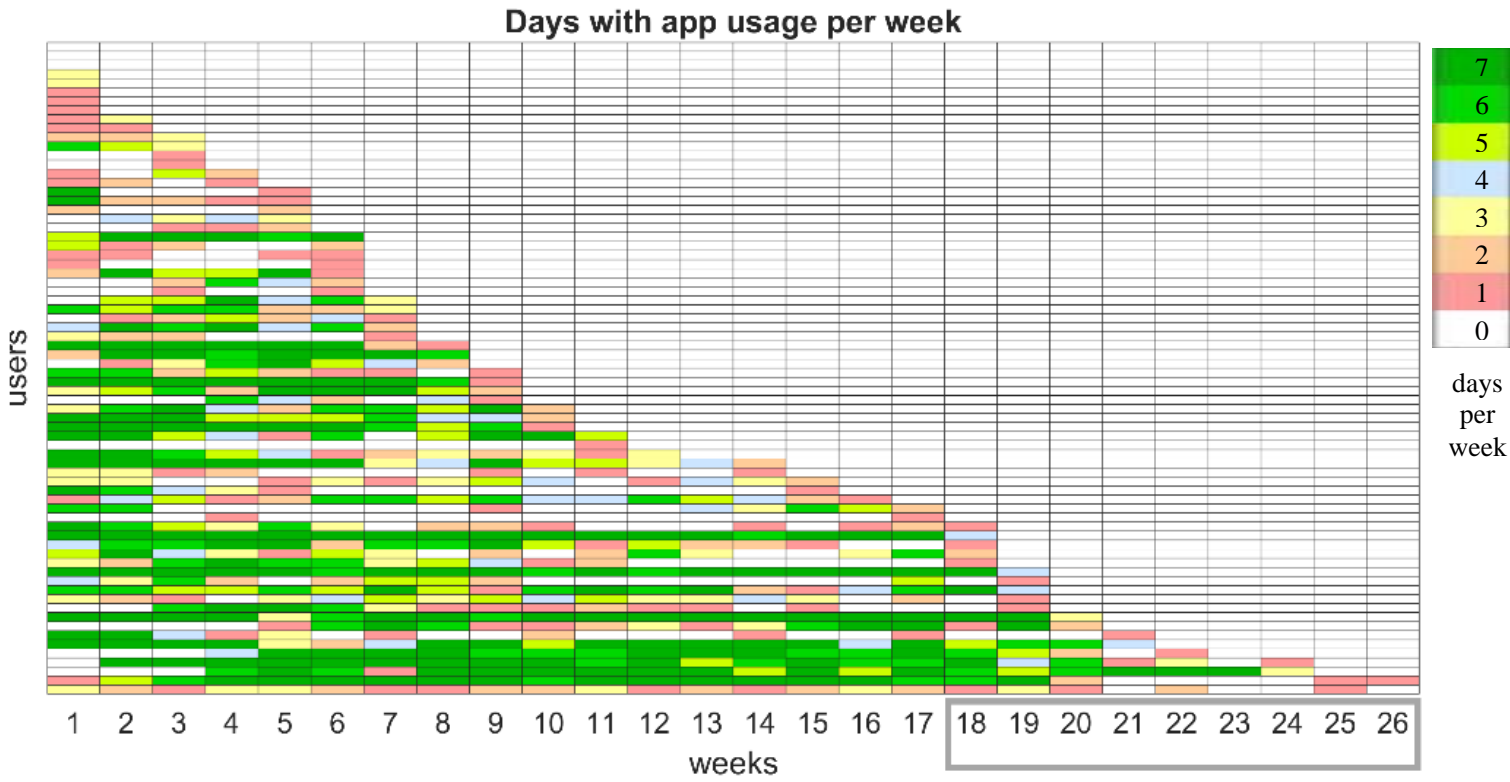

Figure 3. Weekly use, by user, from onboarding up to six months, sorted by use period (the square in grey identifies weeks after the four-month period of use requested by the study) 

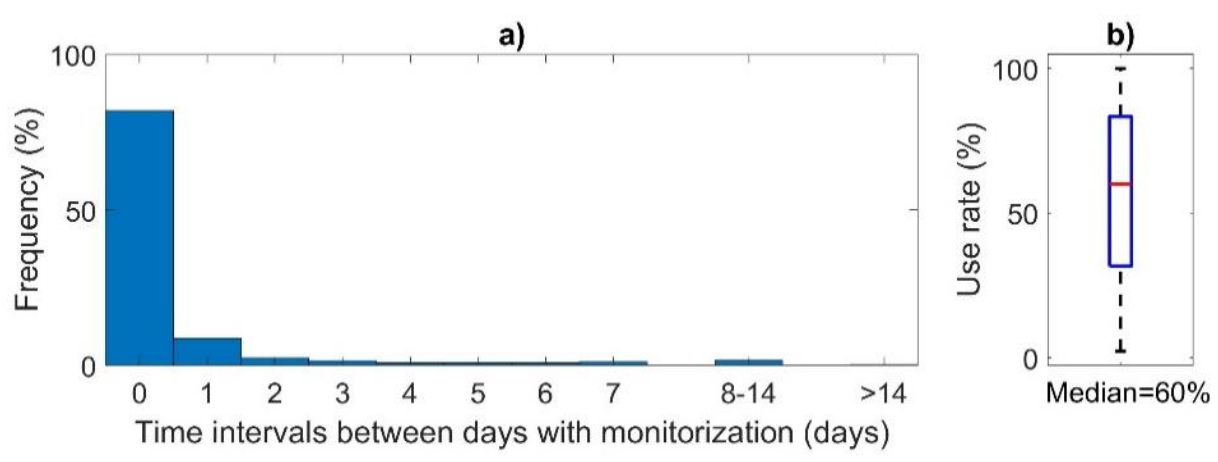

Figure 4. Panel a) Time intervals between days with monitorization (all users); Panel b) usage rate As usual in box and whiskers plot, the central box is limited by the first and third quartiles, with the median marked in red, and the vertical dashed lines reaching the minimum and maximum values of the distribution

Table 1. Description of medication inserted on the App using the "Add medication" option

\begin{tabular}{lllll}
\hline Type of medication & Entries & Uses & Min-Max per user & Mean[sd] per user \\
\hline inhaled & 100 & 57 & $0-8$ & $1.4[1.5]$ \\
pills & 70 & 31 & $0-10$ & $1.0[1.8]$ \\
other (e.g nasal) & 22 & 19 & $0-3$ & $0.3[0.6]$ \\
total & 192 & 64 & $0-15$ & $2.7[3.0]$ \\
\hline
\end{tabular}

Table 2. Number of questionnaires answered in the App

\begin{tabular}{lllll}
\hline Type of questionnaire & Users & Answers & Min-Max per user & Mean[sd] per user \\
\hline Daily & 58 & 999 & $0-69$ & $13.9[17.1]$ \\
Weekly & 65 & 451 & $0-24$ & $6.3[5.9]$ \\
CARAT & 65 & 184 & $0-12$ & $2.6[2.1] 1$ \\
Total & 66 & 1634 & $0-91$ & $22.7[24.1]$ \\
\hline
\end{tabular}

Table 3. Satisfaction (4-5 rating) with InspirerMundi App

\begin{tabular}{lcc}
\hline Satisfaction with respect to & T2 (n=65) & T3 (n=60) \\
\hline Ease of use & $86 \%$ & $88 \%$ \\
Symptoms monitoring component & $88 \%$ & $87 \%$ \\
Willingness to recommend & $85 \%$ & $83 \%$ \\
Integration of the functionalities & $86 \%$ & $78 \%$ \\
Confidence in the App & $80 \%$ & $83 \%$ \\
Global satisfaction with the App & $82 \%$ & $77 \%$ \\
Adherence to medication component & $75 \%$ & $73 \%$ \\
Willingness to use & $75 \%$ & $63 \%$ \\
Gaming component & $72 \%$ & $63 \%$ \\
Image based component & $58 \%$ & $63 \%$ \\
Perceived impact of app use on medication adherence & $58 \%$ & $50 \%$ \\
Social support component & $43 \%$ & $52 \%$ \\
\hline
\end{tabular}

\section{DISCUSSION}

Most participants used the InspirerMundi App to register both medication and symptoms, and the short time between inclusion and first use, together with the reported high global satisfaction, are positive signs. As patients were enrolled in this study invited by their physicians, it was expected that most would become users. Nevertheless, effective monitoring and IMA promoting will only be possible if users adhere to the app, 
using it frequently and consistently. The user retention rates for Health and Fitness apps were reported in 2012 by Flurrymobile as $47 \%$ for 30 -days and $30 \%$ for 90 -days (Farago, 2012). In 2015, the IMS Institute for Healthcare Informatics noticed 30-day retention rates of 59\% for mHealth apps tracked by the AppScript platform after informal recommendation by healthcare providers(Aitken and Lyle, 2015). They estimated an effect of the provider recommendation around 30\%. More recent data, for apps across all industries, from the Adjust Mobile Benchmarks 2018 Edition (UK, France, Germany, Turkey, Japan, US, Russia, and China data) and 2018/2019 statistics from Localytics (an app analytics company in the USA), reported user 30-day retention rates below 15\% (Adjust, 2018) and an average 30-days rate of $43 \%$ (29\% for the average 90 -days rate) (Perro, 2018), expressing the high variability of users behaviour. Also, the percentage of users who abandon an app after one session - user abandonment - was higher than $70 \%$ in the report by Adjust and higher than $80 \%$ in the one from Localytics (Adjust, 2018; Rodde, 2019). Regarding the user retention after 10 sessions, values from $31 \%$ to $39 \%$ have been reported. In this study, less than 5\% of the users abandoned InspirerMundi after the first use and 65\% used the App for more than 10 days, with values for the 30-day retention rate (considering all users) and a 90-day retention rates higher that the previously reported (70\% and 50\%, respectively). This comparatively high retention rate might be positively influenced by the app recommendation by a heath provider in the context of a clinical study, by the phone calls during the study period and the explicit recommendation to use at least for a four-month period. Although, more than $20 \%$ of the users used the App after the 120 days (approximately 4 months or 18 weeks) required by the study.

The 92\% participants answering to at least one questionnaire is in line with the $85.2 \%$ (out of the 7593 enrolled users) replying to at least one survey in the Asthma Mobile Health Study (Chan et al., 2017). Answering CARAT regularly is required to assess asthma control, which has been fulfilled by around $20 \%$ of the users, while $40 \%$ missed just one questionnaire. Registering the prescribed control medication is essential to take advantage of this App for treatment support and monitoring.

At least one inhaled control medication was registered by 57 (79\%) of the 72 users; thirteen users did not register inhalers that were reported as prescribed by the physician. Most users registered medication intakes in the App in most days with App use. Nevertheless, for adequate IMA quantification, daily use was required. Although more than $80 \%$ of the data were introduced in consecutive days, the usage rate was above $75 \%$ in only $35 \%$ of the users. This indicates irregular monitoring, with sets of consecutive monitored days, followed by intervals of non-use or even leaving. Further analysis regarding the clinical data is required to determine if this is related to changes in the therapeutic plan or health deteriorating/improving cycles, resulting from asthma exacerbations. These results highlight the difficulty in achieving a continuous medication monitoring. As a mitigation strategy, the remaining doses counter can be used to assess all doses taken between verified intakes, comparing with the registered therapeutic plan, if the same inhaler is used, and estimate a more precise IMA rate (Vieira-Marques et al., 2020).

The gamification component is intrinsic to the App use. By performing the medication and symptoms related tasks the users earned points, progressed levels and achieved related badges. Most users (81\%) did not send any Inspirers or Warriors requests and, thus, they did not use the peer support resources at all. The social support component was also the one associated with lower user satisfaction. These features need to be reviewed in future app versions to become more attractive to the user and attain their value as tools that facilitates user engagement, promoting a daily, continuous and persistent app usage, which will allow a more effective IMA measure and support. The use of an app can also be evaluated through the number of actions that are performed by the users. That information is not collected by InspirerMundi, which limits the analysis to those metrics that are based on the information directly introduced by the users. Nevertheless, the main actions (related to scheduled medication and questionnaires) are initiated by the app at fixed times or according to the therapeutic plan, and, thus, the rate of fulfilment with respect to the planned might be a more meaningful approach to evaluate InspirerMundi app usage than the number of actions by itself.

Regarding the inhaler detection tool some important limitations are: 1) requiring the correct registration of the inhaled medication (done by the user), to allow the selection of the adequate template, and 2) requiring a specific position of the inhaler, during image collection, with respect to the specified template (no rotation or position adjustments are possible). Our data included entries from 5 users that were not able to insert adequately the type of medication; errors were related to the incorrect identification of the type of drug (as inhaler, pill or other) and one to the introduction of multiples medications under the same entry. These errors, although infrequent, suggested that the way the therapeutic plan was introduced by the user was not completely clear and should be improved. Moreover, when reviewing the inhaler images, it was also noticeable than even when the medication type was correctly selected as inhalation, some users did not correctly registered the specific 
device (thus no template was selected) and $4 \%$ of the images were associated with a wrong template. The app versions that were used in the study included templates for only 6 types of inhalers, which can also lead to errors especially. The updated version of the app has now 11 types of inhaler templates (Diskus/Accuhaler, Easyhaler, Ellipta, Flutiform, K-haller, NEXThaler, Novolizer/Genuair, Seretaide MDI, Spiromax, Turbohaler, Twisthaler), including almost all inhalers used in Europe in 2019 that have a numeric indication of the remaining dosages. Additionally, the quality of most images was very low, with many not showing the complete or not showing the inhaler at all. Previous usability tests had already shown that registering medication intake using the image-based detection tool was not straightforward and that some participants did not understand that they were supposed to use the smartphone's camera (Ferreira et al., 2019). Based on these results, we modified the inclusion of the therapeutic plan in order to guide the user for the correct selection. The visual identification of the type of inhaler is now the first step when introducing the therapeutic plan; then, a full list of medications on the market that use that type of inhaler is presented. Explanatory videos (for medication registration and intake) were also made available.

\section{CONCLUSIONS}

The InspirerMundi app combines features of inhaler usage detection and disease monitoring allowing a reliable and ubiquitous collection of patients' health state and adherence to treatment, using only smartphone embedded sensors. To our knowledge there is no other available solution that allows an image-based objective measurement of inhaled medication adherence. In general, the users started to use the app features right after installation. Use patterns and retention rates indicate that this App can be well accepted among patients, at least if its use is suggested by a specialist. The data retrieved from the mInspirers study allowed to launch an updated version of the App, with improvements in the introduction of the therapeutic plan, currently available at the PlayStore (October 2019). Additional enhancements are required, specially regarding gamification and social components, in order to further improve user engagement.

\section{ACKNOWLEDGEMENT}

This work is partially supported by project NORTE-01-0247-FEDER-033275, financed by the North Portugal Regional Operational Programme (NORTE 2020), under the PORTUGAL 2020 Partnership Agreement, and through the European Regional Development Fund (ERDF). AIRDOC is with ITEA 316040 PHE - Personal Health Empowerment project consortium. R. Almeida is supported through the operation POCI-01-0145-FEDER-029130 (mINSPIRERS- mHealth to measure and improve adherence to medication in chronic obstructive respiratory diseases - generalisation and evaluation of gamification, peer support and advanced image processing technologies) funded by the COMPETE2020 and by National Funds through FCT (Fundação para a Ciência e a Tecnologia).

\section{REFERENCES}

Adjust (2018) MOBILE BENCHMARKS 2018.

Aitken, M. and Lyle, J. (2015) Use, Evidence and Remaining Barriers to Mainstream Acceptance Patient Adoption of mHealth. Available at: www.theimsinstitute.org.

Antoniu, S. A. (2010) 'Adherence to inhaled therapy in COPD: effects on survival and exacerbations', Expert Review of Pharmacoeconomics \& Outcomes Research. Taylor \& Francis, 10(2), pp. 115-117. doi: 10.1586/erp.10.10.

Bender, B. G. (2016) 'Nonadherence to Asthma Treatment: Getting Unstuck.', The journal of allergy and clinical immunology. In practice. Elsevier, 4(5), pp. 849-51. doi: 10.1016/j.jaip.2016.07.007.

Bousquet, J. et al. (2017) 'Realising the potential of mHealth to improve asthma and allergy care: How to shape the future', European Respiratory Journal, 49(5). doi: 10.1183/13993003.00447-2017.

van Boven, J. F. M. et al. (2017) 'Urging Europe to put non-adherence to inhaled respiratory medication higher on the policy agenda: a report from the First European Congress on Adherence to Therapy', The European respiratory journal. doi: 10.1183/13993003.00076-2017. 
Chan, A. H. Y. et al. (2015) 'Using Electronic Monitoring Devices to Measure Inhaler Adherence: A Practical Guide for Clinicians', Journal of Allergy and Clinical Immunology: In Practice. Elsevier Inc, 3(3), pp. 335-349.e5. doi: 10.1016/j.jaip.2015.01.024.

Chan, Y.-F. F. Y. et al. (2017) 'The Asthma Mobile Health Study, a large-scale clinical observational study using ResearchKit', Nature Biotechnology. Nature Publishing Group, 35(4), pp. 354-362. doi: 10.1038/nbt.3826.

DeSmet, A. et al. (2014) 'A meta-analysis of serious digital games for healthy lifestyle promotion', Preventive Medicine. Academic Press, pp. 95-107. doi: 10.1016/j.ypmed.2014.08.026.

Deterding, S. et al. (2011) 'Gamification: Using game design elements in non-gaming contexts', in Conference on Human Factors in Computing Systems - Proceedings. New York, New York, USA: ACM Press, pp. 2425-2428. doi: 10.1145/1979742.1979575.

Doz, M. et al. (2013) 'The association between asthma control, health care costs, and quality of life in France and Spain', BMC Pulmonary Medicine. BioMed Central, 13(1), p. 15. doi: 10.1186/1471-2466-13-15.

Engelkes, M. et al. (2015) 'Medication adherence and the risk of severe asthma exacerbations: a systematic review.', The European respiratory journal. European Respiratory Society, 45(2), pp. 396-407. doi: 10.1183/09031936.00075614.

Farago, P. (2012) App Engagement: The Matrix Reloaded.

Ferreira, A. et al. (2019) 'How Inspiring is your App? A usability take on an App for asthma medication adherence', in 11 th International Conference on e-Health (in press).

GINA (2020) 'GLOBAL STRATEGY FOR ASTHMA MANAGEMENT AND PREVENTION'. doi: 10.15036/arerugi.45.792.

Haase, J., Farris, K. B. and Dorsch, M. P. (2017) 'Mobile Applications to Improve Medication Adherence', Telemedicine and e-Health. Mary Ann Liebert, Inc. 140 Huguenot Street, 3rd Floor New Rochelle, NY 10801 USA, 23(2), pp. 75-79. doi: 10.1089/tmj.2015.0227.

InspirerMundi (2020) YouTube Channel - InspirerMundi App. Available at: https://www.youtube.com/channel/UCCRiXqsttKoHiEGbbykLHxQ.

Jácome, C. et al. (2019) 'Patient-Physician Discordance In The Assessment Of Adherence To Inhaled Medication', in EMJ Allergol Immunol.

Kosse, R. C. et al. (2017) 'mHealth intervention to support asthma self- management in adolescents : the ADAPT study', pp. 571-578.

Marcano Belisario, J. S. J. S. et al. (2013) 'Smartphone and tablet self management apps for asthma.', in Marcano Belisario, J. S. (ed.) The Cochrane database of systematic reviews. Chichester, UK: John Wiley \& Sons, Ltd, p. CD010013. doi: 10.1002/14651858.CD010013.pub2.

Miller, L. et al. (2017) 'Mobile Technology Interventions for Asthma Self-Management: Systematic Review and Meta-Analysis', JMIR mHealth and uHealth. JMIR Publications Inc., 5(5), p. e57. doi: 10.2196/mhealth.7168.

Morton, R. W. et al. (2017) 'STAAR: a randomised controlled trial of electronic adherence monitoring with reminder alarms and feedback to improve clinical outcomes for children with asthma', Thorax, 72(4), pp. 347-354. doi: 10.1136/thoraxjnl-2015-208171.

Ose, D. et al. (2012) 'Let's talk about medication: Concordance in rating medication adherence among multimorbid patients and their general practitioners', Patient Preference and Adherence. Dove Press, 6, pp. 839-845. doi: 10.2147/PPA.S35498.

Perro, J. (2018) Mobile Apps: What's A Good Retention Rate? Available at: http://info.localytics.com/blog/mobile-appswhats-a-good-retention-rate (Accessed: 21 August 2019).

Rodde, T. (2019) 25\% of Users Abandon Apps After One Use, localytics. Available at: http://info.localytics.com/blog/25of-users-abandon-apps-after-one-use (Accessed: 21 August 2019).

Sardi, L., Idri, A. and Fernández-Alemán, J. L. (2017) 'A systematic review of gamification in e-Health', Journal of Biomedical Informatics. Academic Press, pp. 31-48. doi: 10.1016/j.jbi.2017.05.011.

Sheffield, E. R. S. (2017) Forum of International Respiratory Societies. The Global Impact of Respiratory Disease- Second Edition, Forum of International Respiratory Societies. Sheffield: European Respiratory Society. Available at: https://www.fi (Accessed: 15 April 2020).

Tinschert, P. et al. (2017) 'The Potential of Mobile Apps for Improving Asthma Self-Management: A Review of Publicly Available and Well-Adopted Asthma Apps', JMIR mHealth and uHealth. JMIR Publications Inc., 5(8), p. e113. doi: 10.2196/mhealth.7177.

Vieira-Marques, P. et al. (2020) 'Combined Image-Based Approach for Monitoring the Adherence to Inhaled Medications', in, pp. 1399-1404. doi: 10.1007/978-3-030-31635-8_171. 